\title{
Método del caso aplicado a la asignatura de Ingeniería del Riego del grado de Ingeniería Agroalimentaria y del Medio Rural.
}

\author{
Carmen Virginia Palau ${ }^{a}$, Jaime Arviza ${ }^{b}$, Iban Balbastre ${ }^{c}$
}

aPDI, Dpto. Ingeniería Rural, Universitat Politècnica de València, virpaes@agf.upv.es, bPDI Dpto.

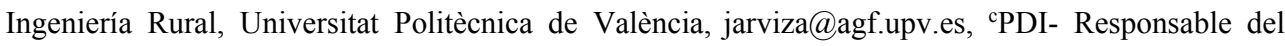
Laboratorio de Hidráulica y Riegos de la Universitat Politècnica de València, ibbalpe@agf.upv.es.

\begin{abstract}
This paper presents the planning and results obtained in the subject of Irrigation Engineering within the degree of Rural and Agrifood Engineering after the application of active learning- teaching methodologies for two academic years. Using the case study method students face real situations that reflect and solve under the guidance of the teacher. The evaluation is done through a virtual portfolio of each study and the resolution of a case in reduced groups and the drafting of a final report. The results show a dynamic teaching methodology that encourages students to reflect and learn about real situations. Although certain improvements are spelled out in the methodology presented to be planned in future courses.
\end{abstract}

Keywords: active learning, case study, collaborative learning , evaluation.

\begin{abstract}
Resumen
Esta comunicación presenta la planificación y resultados obtenidos en asignatura de Ingeniería del Riego dentro del grado de Ingeniería Agroalimentaria y del Medio Rural tras la aplicación de metodologías activas de aprendizaje- enseñanza durante dos cursos académicos. Para ello, mediante el método del caso, los alumnos afrontan situaciones reales que reflexionan y resuelven bajo las directrices del profesor. La evaluación se realiza a través de un portafolio virtual de cada estudio y con la resolución de un caso en grupo y la redacción de un informe final. Los resultados muestran una metodología docente dinámica que motiva al alumno a reflexionar y aprender sobre situaciones reales, aunque se puntualizan ciertas mejoras en la metodología presentada que se planificaran en próximos cursos.
\end{abstract}

Palabras clave: metodología activa, caso, diseño, aprendizaje colaborativo, evaluación.

\section{Introducción}

El proceso de Bolonia (1999) ha propiciado cambios importantes en el entorno de la educación superior en Europa. En este sentido, se han experimentado numerosas transformaciones en los modelos formativos y nuevas propuestas en innovación docente. 
Una de estas novedades es la introducción de metodologías activas en el aula que propicien la reflexión del alumno sobre lo que hace, y no meramente, que acumule los conocimientos transferidos por el profesor durante una lección magistral.

Las metodologías activas son técnicas de enseñanza y aprendizaje centradas en el estudiante como elemento conductor de su propio aprendizaje. El profesor acompaña, guía, planifica, motiva, evalúa al estudiante en este proceso, le enseña a aprender a aprender (Fernández March, 2006).

De esta forma, en la asignatura de Ingeniería del Riego del grado en Ingeniería Agroalimentaria y del Medio Rural de la Universitat Politècnica de València se lleva a cabo una combinación de metodologías de aprendizaje que fomentan el trabajo autodirigido del alumno, haciéndolo responsable de su propio aprendizaje.

La asignatura se diseñó y planificó con una serie de actividades de aprendizaje-enseñanza basadas en la metodología del caso (MdC), descrita en el programa Eragin de la Universidad del País Vasco, que aproximan al estudiante a la realidad profesional para lograr la adquisición de un conocimiento más profundo y constructivo. Además, en ellos se experimenta una mayor motivación y entusiasmo hacia la temática de la asignatura.

Como indica De Miguel en 2005, el estudio de casos permite un análisis intensivo y completo de un hecho, problema o suceso real con la finalidad de resolverlo, contrastar datos, reflexionar $y$, en ocasiones, entrenarse en posibles soluciones alternativas.

\section{Objetivos}

El objetivo principal de este artículo es presentar la experiencia docente llevada a cabo desde 2014 con los alumnos de grado de Ingeniería Agroalimentaria y del Medio Rural, analizar los resultados obtenidos con el cambio de metodología de trabajo y valorar si su implantación aumenta la implicación y motivación del alumnado, así como su grado de aprendizaje.

Los indicadores que permiten valorar la metodología docente empleada en la asignatura serán los resultados obtenidos por los estudiantes, el análisis de las encuestas de opinión realizadas a los estudiantes al finalizar el curso, y las entrevistas y sugerencias realizadas por ellos a los profesores durante los diferentes cursos.

\section{Desarrollo de la innovación educativa}

\subsection{Contexto y diseño de las actividades de enseñanza-aprendizaje.}

La asignatura cuatrimestral de Ingeniería del Riego (4.5 ETCS) del grado en Ingeniería Agroalimentaria y del Medio Rural es obligatoria de tercer curso, y desde su inicio en el curso académico 2013-2014, se ha planificado con una combinación de metodologías de enseñanza-aprendizaje basadas en lecciones magistrales donde se presentan los contenidos teóricos y de mayor dificultad de comprensión. Estas lecciones magistrales de teoría están conducidas por los profesores, de manera estructurada y clara, potenciando la participación 
del alumnado mediante preguntas y actividades diversas intercaladas. Este tipo de metodología representa aproximadamente el $40 \%$ de la actividad docente planificada en la guía docente y el $15 \%$ son prácticas de campo y laboratorio.

El 45\% de las actividades se organizan con el método del caso como vehículo conductor de la formación del alumno. Así pues, en esta metodología activa se plantean sucesivos casos bien elaborados e interrelacionados con los fundamentos teóricos vistos durante las clases teóricas, donde el alumno activo, reflexiona, discute y analiza las posibles soluciones al caso expuesto.

El estudio de casos en la asignatura está distribuido por unidades didácticas dentro de un proceso completo de diseño de un sistema de riego a presión. De esta forma, partiendo de la forma de riego en una parcela con un cultivo en producción se avanza aguas arriba hasta el punto de origen o cabezal de riego desde donde parte el agua.

Así pues, la materia organizada en cinco unidades didácticas permite el estudio de diferentes casos, interligados entre sí, con la finalidad de diseñar de forma adecuada un sistema completo de riego a presión (Fig. 1).

UNIDADES DIDÁCTICAS

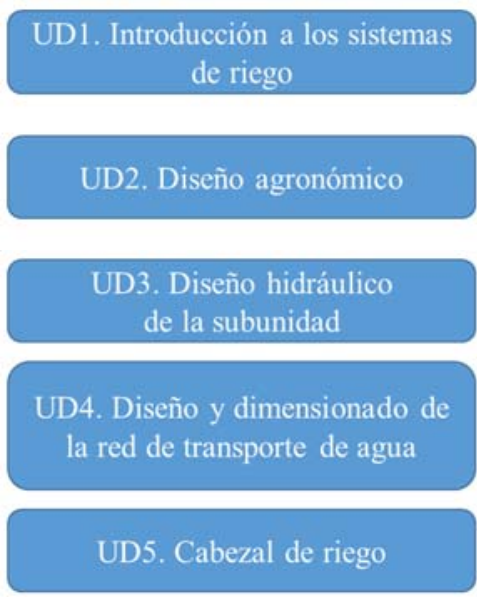

\section{CASO}

Estudio de casos planteados en una misma parcela con un único cultivo en producción p.e. cultivo de caqui en una zona regable de Picassent

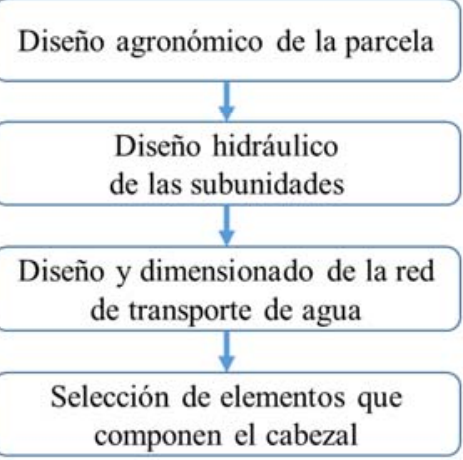

Fig. 1 Distribución de casos de estudio.

\subsection{Planificación de casos de estudio.}

Uno de los aspectos más importantes de la planificación de la metodología de aprendizajeenseñanza es que los alumnos perciban las actividades que se les proponen y el hilo conductor que las une.

Como se ha comentado, las unidades didácticas están interrelacionadas, y es de suma importancia que el profesor exponga las directrices a seguir en cada caso.

(cc) EY-NC-ND 2016, Universitat Politècnica de València

Congreso In-Red (2016) 
De esta forma, el estudiante afronta un problema dentro de un escenario real que debe estructurar, y esforzarse, por encontrar soluciones para resolverlo (Johnson et al. 2000).

En consecuencia, el problema expuesto en cada curso académico es una zona regable real común con unos datos de partida buscados en diferentes fuentes y establecidos para su resolución. También, se emplaza y digitaliza la zona de estudio para poder diseñar y plasmar correctamente las posibles alternativas.

De este escenario común descrito derivan los diversos casos de estudio planteados.

\subsubsection{Caso: Diseño agronómico de la parcela.}

Se plantea para su resolución analítica por parejas o mediante el uso de herramientas informáticas desarrolladas en entorno Excel y programadas en Visual Basic (DISEÑO AGRONÓMICO RL).

- Buscar y ordenar la información de partida necesaria para el diseño agronómico.

- Estimar sus necesidades de riego considerando los parámetros climáticos, de uniformidad de riego, eficiencia de aplicación y fracción de lavado.

- Concretar la disposición del sistema de riego en parcela considerando los criterios de localización del agua en la zona radicular y de solape entre bulbos húmedos.

\subsubsection{Caso: Diseño hidráulico de una subunidad de riego.}

Bajo la premisa de una buena uniformidad de riego, se plantea este caso, para diseñar y dimensionar el sistema de tuberías dispuestas en la subunidad.

Asimismo, su resolución se realiza en diferentes sesiones de forma analítica y mediante un software DIMSUB programado para el diseño de subunidades. Como recurso complementario para la resolución de los casos, se ofrecen diferentes materiales multimedia del uso de esta aplicación (Fig. 2).

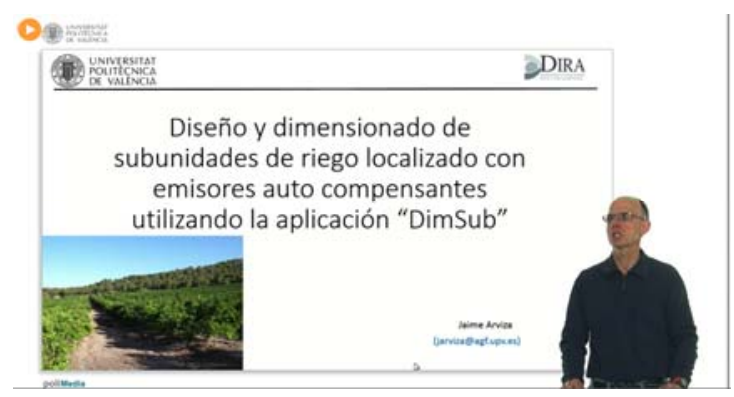

Fig. 2 Material multimedia de apoyo en la resolución de casos.

\subsubsection{Caso: Dimensionado de la red de transporte de agua y evaluación del funcionamiento de la red en EPANET.}

Los alumnos deberán aplicar los procedimientos de diseño y dimensionado de las infraestructuras hidráulicas, a partir de los conocimientos adquiridos en la asignatura, en base 
a criterios funcionales y de optimización técnico económica con un aprovechamiento eficiente de recursos disponibles.

Los alumnos abordan el diseño y dimensionado de la red de transporte, conjunto de tuberías que conectan cada subunidad con el punto de alimentación utilizando la aplicación RGW2015. Para cada hipótesis de partida deben interpretar los resultados y con la citada aplicación exportar los resultados para el análisis de la respuesta hidráulica de la red mediante EPANET.

\subsubsection{Caso: Selección de elementos y diseño en planta del cabezal de riego.}

Para finalizar la materia, se completará con la elección de los elementos necesarios en el cabezal de riego localizado y su ubicación en planta de acuerdo a las características técnicas extraídas por el alumno de catálogos de fabricantes.

\subsection{Evaluación de la actividad.}

La metodología del caso finaliza evaluando los conocimientos adquiridos durante los diferentes estudios planteados con un portafolio virtual que el alumno va depositando y acumulando tras cada sesión.

Y como trabajo no presencial ¿qué mejor manera que plantear otro caso?. Los alumnos mediante la estrategia de aprendizaje colaborativo en grupos reducidos, con los recursos vistos y las herramientas utilizadas, lo resuelven escribiendo un informe de la solución al problema planteado.

Tabla 2. Metodologías, instrumentos para la evaluación y pesos dentro de la materia.

\begin{tabular}{lll}
\hline Metodología docente & Instrumentos de evaluación & Peso calificación \\
\hline Método del caso & Portafolio virtual de casos planteados & $15 \%$ \\
\hline Aprendizaje colaborativo & Trabajo académico de resolución en grupo & $15 \%$ \\
\hline Expositivo & Prueba escrita de respuesta abierta & $60 \%$ \\
\hline Portafolio de prácticas & $\begin{array}{l}\text { Organización y desarrollo del portafolio } \\
\text { de tareas de laboratorio y campo }\end{array}$ & $10 \%$ \\
\hline
\end{tabular}

\section{Resultados de la aplicación de las metodologías activas.}

Los resultados desde el curso académico 2012-2013 donde se pusieron en marcha el estudio de casos en la asignatura de Ingeniería del Riego han sido bastante satisfactorios. En el primer curso se advirtió cierta descoordinación entre teoría de aula y seminarios donde se estudiaban los casos, pero en general, el método del caso motiva y aproxima a la práctica profesional al estudiante. 
La valoración de los alumnos tras dos cursos académicos de Ingeniería del Riego se resume en la siguiente Tabla 3 .

Tabla 3. Resultados de las encuestas de opinión del alumnado.

\begin{tabular}{ccc}
\hline DIMENSION & Curso 2013-2014 & Curso 2014-2015 \\
\hline $\begin{array}{c}\text { Motivación/interacción } \\
\text { estudiante-materia }\end{array}$ & 7 & 8 \\
\hline Metodología empleada & 7 & 6 \\
\hline Organización y planificación & 7 & 7 \\
\hline Recursos y materiales utilizados & 6 & 7,2 \\
\hline
\end{tabular}

Tanto la tasa de éxito que estuvo en torno al $90 \%$ en ambos cursos, como la tasa de rendimiento aproximadamente del 85\% manifestó los buenos resultados logrados por los alumnos en la evaluación de sus conocimientos en la materia.

Así mismo, se realizaron entrevistas a los alumnos para conocer su opinión acerca de la metodología empleada, y las respuestas fueron positivas con algunas aportaciones interesantes que se han ido implementando en el siguiente curso como mejora docente.

Los alumnos manifestaron su descontento en la planificación inicial de algunos casos y también en la carga de trabajo que suponía su resolución atendiendo a los cortos plazos establecidos. En este sentido, durante este curso se han ampliado los plazos de entrega y los alumnos están elaborando su propio portafolio virtual con cada uno de los casos estudiados, del que se evaluarán una parte al azar de los mismos.

Finalmente, mostraron una opinión positiva hacia los nuevos recursos multimedia empleados, hacia las herramientas de diseño utilizadas y hacia las actividades desarrolladas, indicando que les había dado un visión global del problema coherente y cercana a una futura práctica profesional.

\section{Conclusiones}

En esta comunicación se muestra la experiencia llevada a cabo en la asignatura de Ingeniería del Riego del grado de Ingeniería Agroalimentaria y del Medio Rural con la utilización del método del caso como vehículo para la formación del estudiante universitario.

Los casos reales desarrollados parten siempre de un mismo escenario y van resolviéndose las diferentes fases del diseño de un sistema de riego localizado, lo que permite al alumno visualizar en conjunto y con un nexo en común que es la misma zona regable, todos los resultados de aprendizaje establecidos .

Investigaciones futuras permitirán comparar la metodología docente presentada con la desarrollada tradicionalmente por los profesores mediante sistemas convencionales. Adicionalmente, se programará alguna actividad complementaria basada en esta metodología que permita enseñar y evaluar la competencia transversal asignada a esta asignatura.

(cc) EY-NC-ND 2016, Universitat Politècnica de València

Congreso IN-RED (2016) 
Finalmente, los resultados obtenidos durante los dos cursos académicos han sido satisfactorios y el grado de implicación y rendimiento de los alumnos alto. También, se concluye con una serie de propuestas de mejora que se están implementando en el presente curso académico y que en trabajos posteriores se conocerán sus frutos.

\section{Referencias}

DE MIGUEL (2005). Modalidades de enseñanza centradas en el desarrollo de competencias: orientaciones para promover el cambio metodológico en el Espacio Europeo de Educación Superior. Proyecto EA2005-0118. Ed. Universidad de Oviedo. ISBN 978-84-8317-546-0.

FERNADEZ MARCH, A (2006). Metodologías activas para la formación en competencias. Educatio siglo XXI, Vol. 24, pp. 35 -56.

JOHNSON, D.W., JOHNSON,R.T.,and SMITH, K.A., (2000). Active Learning: Cooperation in the College Classroom. Interaction Book, Edina, MN.

MINISTROS EUROPEOS DE EDUCACIÓN (1999). Declaración de Bolonia. Espacio Europeo de Educación Superior.

UNIVERSITAT POLITÈCNICA DE VALENCIA. Proyecto Institucional sobre Competencias Trasnversales.

\section{Libro electrónico}

GUISASOLA, J; GARMENDIA, M. (2014) El programa ERAGIN de formación en metodologíasactivas de la UPV/EHU. Aprendizaje basado en problemas, proyectos y casos: diseño e implementación de experiencias en la universidad. Eds. Universitad del Pais Vasco.

\section{Software}

DISEÑO AGRONÓMICO RL (2015). J Arviza.

DIMSUB, (2015) J Arviza.

RGW2015 (2015), J.Arviza

\section{Páginas web}

PORTAL UNIVERSIDAT POLITECNICA DE VALENCIA < http://competenciast.webs.upv.es/>.

UNIVERSIDAD DEL PAIS VASCO, Programa Eragin $<$ http://www.ehu.eus/es/web/sae-helaz/eraginirakaskuntza-metodologia-aktiboak>

ESCUELA TÉCNICA SUPERIOR DE INGENIERÍA AGRONÓMICA Y DEL MEDIO NATURAL (2013). Grado en Ingeniería Agroalimentaria y del Medio Rural

$<$ https://www.upv.es/titulaciones/GIAMR/indexc.html>

(c)) EY-NC-ND 2016, Universitat Politècnica de València

Congreso In-Red (2016) 